\title{
Fluid Deformation of Serial Structural MRI for Low-Grade Glioma Growth Analysis
}

\author{
Bernard Cena ${ }^{1}$, Nick Fox ${ }^{1}$, and Jeremy Rees ${ }^{2}$ \\ ${ }^{1}$ Dementia Research Group, Institute of Neurology, University College London, \\ 8-11 Queen Square, London WC1N 3BG, UK \\ \{bcena,nfox\}@dementia.ion.ucl.ac.uk \\ 2 Department of Molecular Neuroscience, Institute of Neurology, National Hospital \\ for Neurology and Neurosurgery, London, UK
}

\begin{abstract}
We apply fluid model non-rigid registration of serial structural T1 weighted MR images to obtain deformation-based measurements of low-grade glioma growth. Preliminary experimental results from ten patients show that measurements of tumour regions, together with visual inspection, provide insight into glioma growth characteristics.
\end{abstract}

\section{Introduction and Method}

Gliomas are the most common primary brain tumours. The management of lowgrade gliomas (LGG) is difficult because they grow slowly, infiltrate diffusely and patient survival time is highly variable. Most cases of LGG progress to high-grade glioma (HGG) by a process of malignant transformation, which is an unpredictable event in the individual patient [1]. This study aims to measure LGG growth using serial T1-weighted MR imaging. Our imaging protocol includes structural T1 and fast spin echo T2, fFLAIR, sequences. Ten patients were chosen from a glioma imaging project of whom five were clinically and radiologically stable (defined as non-transforming), the others progressed clinically or developed a new area of gadolinium enhancement (transforming). All patients have had biopsies to confirm the diagnosis and have been imaged at 6 monthly intervals with a minimum of 4 studies.

For each study, the tumour region was roughly segmented by simple semiautomated thresholding on a fFLAIR image to provide a region of interest (ROI) and then co-registered with the corresponding T1 image. The fFLAIR image provides good contrast for anatomy affected by the tumour, but suffers from poor axial resolution ( $6 \mathrm{~mm}$ slices). For each patient, all $\mathrm{T} 1$ studies were resampled to cubic voxels $\left(2 \mathrm{~mm}^{3}\right)$ and rigidly registered (with rescaling) to baseline $\mathrm{T} 1$. For each consecutive pair of studies, the later study was non-rigidly registered to the earlier using a fluid model deformation [2]. Tumour growth was assessed by integration of the Jacobian determinant of the deformation field at all voxels over the union of tumour regions at all time points for each patient. Malignant transformation was assessed by connected component analysis of the top $5 \%$ of Jacobians inside the composite ROI. 


\section{Results and Discussion}

Table 1 shows growth rates in $\mathrm{mm}^{3}$ for each subject adjusted for time (6 month interval). These growth rates correspond to the total contribution of connected components of the top 5\% (empirical choice) of Jacobian values. Transforming patients $(\mathrm{T})$ show steady or accelerating expansion of the tumour. The data for the non-transforming (NT) group suggests static or steady growth.

Table 1. Growth rates of highest growth clusters for transforming (T) and nontransforming $(\mathrm{NT})$ subjects in $\mathrm{mm}^{3}$ adjusted for time interval. $\dagger$ Patient $\mathrm{T} 1$ had chemotherapy between time points 5 and 6 .

\begin{tabular}{|c|c|c|}
\hline interval & \begin{tabular}{lllll|} 
T1 & T2 & T3 & T4 & T5 \\
\end{tabular} & NT1 NT2 NT3 NT4 NT5 \\
\hline $1 \rightarrow 2$ & $\begin{array}{lllll}1.14 & 3.42 & 1.30 & 1.48 & 1.22\end{array}$ & $\begin{array}{|lllll|}0.51 & 3.21 & 1.00 & 1.79 & 0.64 \\
\end{array}$ \\
\hline $2 \rightarrow 3$ & 1.403 .494 .331 .302 .74 & $\begin{array}{lllll}0.51 & 1.38 & 2.36 & 3.40 & 1.1\end{array}$ \\
\hline $3 \rightarrow 4$ & 1.063 .344 .452 .595 .58 & $\begin{array}{lllll}0.36 & 1.68 & 2.87 & 1.78 & 0.9\end{array}$ \\
\hline $4 \rightarrow 5$ & 2.915 .06 & $\begin{array}{lllll}0.80 & 2.51 & 3.25 & 1.13 & 0 .\end{array}$ \\
\hline $5 \rightarrow 6$ & 0.167 .80 & 0.36 \\
\hline
\end{tabular}

We chose a very high degree of freedom (DOF) non-rigid registration algorithm based on a fluid model [2] to avoid making assumptions about the unknown glioma growth characteristics. The fluid model enables measurement of both displacing and, to some degree, infiltrating tumour growth, but suffers from slow convergence in areas of large displacement. We monitored the convergence of all registrations by visual analysis of transformed images.

The pattern of glioma growth is unique for every patient. Analysis of the highest growth areas has potential for predicting foci of malignant transformation. We plan to introduce a medium DOF registration between the rigid and fluid stages in order to normalise large scale deformations and achieve faster and better convergence of the fluid stage. Correlation of these preliminary results with perfusion and diffusion studies, and more sophisticated analysis of the deformation fields, is expected to provide further information about the process of LGG to HGG transformation.

Currently, the regional measurements combined with visual analysis of the deformations provide valuable clinical insight into glioma growth progression.

Acknowledgment. The authors thank Mr. Chris Benton for the MRI data.

\section{References}

1. Rees, J.: Advances in magnetic resonance imageing of brain tumours. Current Opinion in Neurology 16 (2003) 643-650

2. Freeborough, P.A., Fox, N.C.: Modeling brain deformation in Alzheimer Disease by fluid registration of serial 3D MR images. J. Comput. Assist. Tomogr. 22 (1998) $838-843$ 